\title{
A FORMAÇÃO DOCENTE PARA O ENSINO DE ESPANHOL POR UMA ABORDAGEM INTERCULTURAL NO ENSINO
}

\author{
Ana Paula do Nascimento VELÁSQUEZ ${ }^{1}$ \\ Jéssica Cardoso PAES ${ }^{2}$
}

\begin{abstract}
RESUMO
Este trabalho discute o aspecto intercultural na formação docente inicial para o ensino de língua espanhola. Partimos do pressuposto de que a formação docente apresenta uma lacuna em seus desenhos curriculares com relação a diversidade cultural hispanófona promovendo uma aprendizagem dos aspectos linguísticos desarticulada de práticas culturais. Conforme os Parâmetros Curriculares Nacionais (Brasil, 2000), a aprendizagem de uma língua estrangeira deve ser entendida como fonte de ampliação dos horizontes culturais que levam o aluno a refletir, pois conhecer outras culturas e outras formas de lidar com a realidade capacita $\mathrm{o}$ aluno a pensar não somente sobre a sua própria cultura mas lhe dar condições de confrontar semelhanças e diferenças entre a sua cultura em relação à outras culturas. Para tanto, nos apoiamos em Irineu (2014) por tratar da invisibilidade da América Latina em detrimento da relevância dada à variedade linguística e cultural peninsular na formação do professor de língua espanhola e Fleuri (2003) cujos estudos defendem a interculturalidade no ensino com o objetivo de promover a aproximação entre os povos. Inicialmente desenvolvemos a pesquisa analisando o desenho curricular de três instituições de ensino superior na cidade de Belém. Após a análise dos três desenhos curriculares das instituições acima mencionadas constatamos que apenas um desenho apresenta atividade curricular voltada para a abordagem dessa temática, em seguida aplicamos um questionário como instrumento de investigação para seis professores egressos dessas instituições. Como resultado, a pesquisa confirmou que durante a formação docente dos professores investigados a abordagem intercultural não foi privilegiada, ratificando o que afirma Irineu sobre a invisibilidade da cultura latino-americana.
\end{abstract}

Palavras-chave: Ensino. Formação Docente. Interculturalidade.

\begin{abstract}
This work discusses the intercultural aspect in initial teacher training for Spanish language teaching. We star from de assumption that the teacher training presents a gap in his curricular drawings with respect to Hispanic-speaking cultural diversity promoting a learning of the disjointed linguistic aspects of cultural practices. According to the National Curriculum Parameters (Brazil, 2000), the learning of a foreign language should be understood as a source of expansion of the cultural horizons that lead the student to reflect, since knowing other cultures and other ways of dealing with reality enables the student to think not only about their own culture but give them conditions to confront similarities and differences between their culture in relation to other cultures. To that end, we rely on Irineu (2014) for dealing with the invisibility of Latin America to the detriment of the relevance given to the peninsular linguistic and cultural variety in the formation of the Spanish-language teacher and Fleuri (2003) whose studies defend interculturality in teaching with the objective to promote rapprochement between peoples. After analyzing the three curricular designs of the above mentioned institutions, we found that only one drawing presents curricular activity focused on the approach of this subject, then we applied a questionnaire as a research tool for six teachers who graduated from these institutions. As a result, the research confirmed that during the teacher training of the teachers investigated the intercultural approach was not privileged, ratifying what Irineu says about the invisibility of Latin American culture.
\end{abstract}

Keywords: Teaching. Teacher training. Interculturality.

\footnotetext{
1 Mestranda do Programa de Pós-Graduação em Educação e Cultura (PPGEDUC) na Universidade Federal do Pará - Campus Universitário do Tocantins/Cametá. Atualmente é docente efetiva da Universida-
}

de Federal do Pará - Campus 2 Graduanda em Letras Universitário do Baixo To- UFPA. cantins / Abaetetuba.

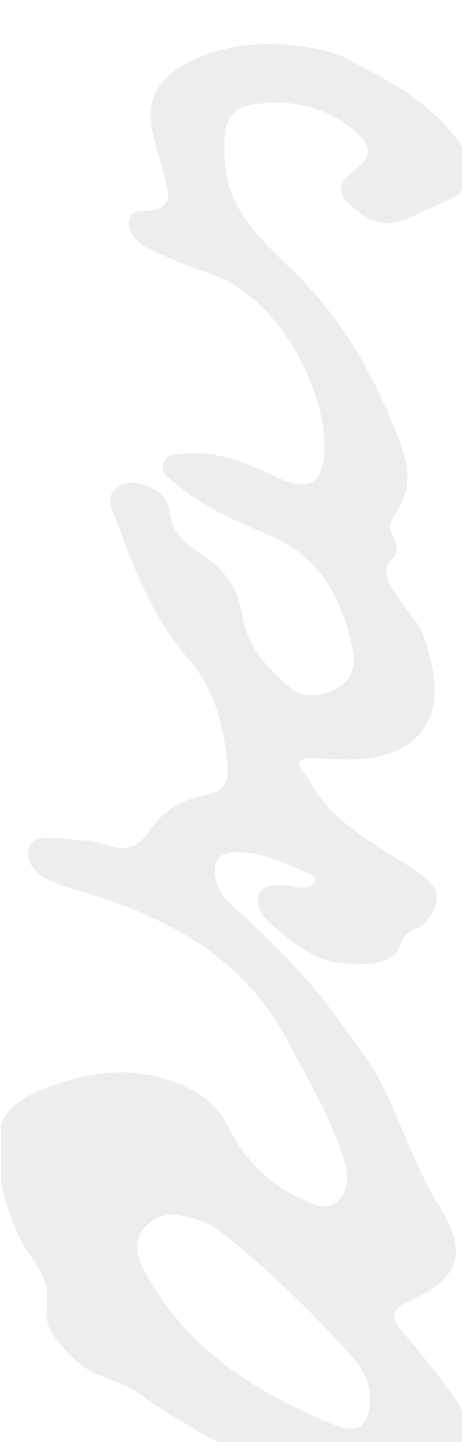




\section{INTRODUÇ̃̃O}

Este artigo apresenta uma discussão no que concerne ao aspecto intercultural na formação docente inicial para o ensino de língua espanhola. Durante um longo período, o espanhol permaneceu no ostracismo no cenário educacional brasileiro, renascendo com a criação do MERCOSUL que trouxe a proposta de integração da região sul do continente americano levando à sanção, alguns anos mais tarde, da Lei Federal $\mathrm{n}^{\mathrm{o}} 11.161 / 2005$ que determinava a oferta obrigatória do ensino desse idioma no Ensino Médio em todas as escolas das redes particular e pública.

Atualmente, a formação de professor para ensinar língua espanhola ocorre em três instituições de ensino superior na cidade de Belém. Iniciou-se na primeira e única universidade particular sediada no Estado do Pará - a Universidade da Amazônia (UNAMA) - em 1996, seguida em um intervalo de seis anos pela Escola Superior Madre Celeste (ESMAC) e cinco anos depois passou a ser ofertado também pela Universidade Federal do Pará (UFPA), no campus de Belém, e posteriormente nos campi de Abaetetuba e Castanhal, municípios da região metropolitana.

Analisando os respectivos Projeto Pedagógico do Curso (PPC) constatamos que somente a ESMAC tem uma atividade curricular denominada Etnicidade e Multiculturalismo que contempla a questão dos deslocamentos de grandes massas populacionais para várias áreas de acolhimento distintas ocasionando o entrecruzamento de culturas, enquanto que nos desenhos curriculares da UNAMA e UFPA - campus de Abaetetuba - não há quaisquer inclinação para a abordagem dessa temática. Após essa análise, selecionamos 06 (seis) professores atuantes no ensino médio em escolas particulares e públicas na condição de egressos dessas instituições de ensino superior para a realização de uma entrevista guiada. A escolha dos investigados esteve motivada, em primeiro plano, pelo tempo de atuação no ensino do espanhol com, no mínimo, dez anos, e, em segundo plano, pelo fato de trabalharem em algumas escolas classificadas no ranking do ENEM em 2015.

Aprender uma língua estrangeira, de acordo com os Parâmetros Curriculares Nacionais (PCNs), tem como objetivo ampliar a visão de mundo do aprendiz, ter acesso à outras culturas, à informação internacional e estabelecer contato com pessoas de diversas nacionalidades. Nesse sentido, podemos afirmar que na aprendizagem está imbrincado um caráter social e político; social porque possibilita ao aprendiz a aquisição de conhecimentos diversificados e político porque viabiliza a comunicação com pessoas de nacionalidades diferentes ao mesmo tempo que oportuniza o ensinamento da cultura materna para os estrangeiros. A esse respeito, Santos (2004, p.16) faz recordar que "ensinar e aprender uma nova língua-cultura deve ser, portanto, um processo em duas vias: da língua-cultura-alvo em relação às línguas-culturas que estão ali em interação e vice-versa". Com essa assertiva, a autora é consciente que os professores são responsáveis por ensinar o que é relativo ao 'outro', porém ressalta que se deve considerar a cultura materna e estabelecer um paralelo, uma relação dialógica, entre a cultura estrangeira e a própria do aprendiz. Mas, afinal, o que é cultura?

[...] a palavra cultura passou a ser utilizada para se referir a tudo o que seja característico sobre o "modo de vida de um povo, de uma comunidade, de uma nação ou de um grupo social - o que veio a ser conhecido como a definição "antropológica". Por outro lado, a palavra também passou a ser utilizada para descrever os "valores compartilhados" de um grupo ou de uma sociedade - o que de certo modo se assemelha à definição antropológica, mas com uma ênfase sociológica maior (HALL, 2016, p. 19).

Do ponto de vista desse estudioso, a relevância sociológica da cultura está para além do registro descontextualizado da maneira como vive um povo. A ênfase reside nas regras para uma interação harmoniosa, tranquila e segura de uma comuni- 
dade e a língua faz parte da realidade social.

No entanto, de acordo com o PCNs " [...] na escola regular (as aulas) passaram a pautar-se, quase sempre, apenas no estudo de formas gramaticais, na memorização de regras e na prioridade da língua escrita, em geral, tudo isso de forma descontextualizada e desvinculada da realidade" (BRASIL, 2000, p.26). Tal prática educativa não considera a relevância do ensino de línguas estrangeiras na educação básica para o estreitamento de relações com outras culturas ao mesmo tempo que desvincula esse aprendizado da formação cidadã do aprendiz para exercício da democracia na sociedade. Soma-se a esse ensino metalinguageiro (o uso da língua para explicar o funcionamento dela mesma) nas escolas à pouca visibilidade dada aos países latino-americanos com relação aos seus aspectos linguístico e cultural que se constituem como fontes de enriquecimento de conhecimento.

A respeito desse quase apagamento da América Latina, Irineu (2014) retrata que o olhar lançado sobre essa região é de um conjunto de países com paisagens e indivíduos exóticos, diferentes dos habituais cenários e personagens europeus, tão massivamente retratados nos livros didáticos e que despertam mais o interesse dos alunos em contraste com a ideia de cultura primitiva, tradicional, atrasada e subdesenvolvida dos países latino-americanos. Essa ideia preconceituosa acaba por não contribuir com a abertura de um diálogo entre as culturas hispanófonas e brasileiras e consequentemente dificultar o ensino do espanhol por meio de uma abordagem intercultural.

Para Fleuri (2003), a abordagem intercultural viabiliza não apenas o conhecimento e a valorização de diversas culturas, mas a aproximação a medida em que se identificam as semelhanças e os distanciamentos entre elas. No caso do espanhol, construir pontes entre a cultura materna e a da língua-alvo contribui, sobretudo, para a construção da identidade latino-americana do Brasil que durante algum tempo não era reconhecido como parte indissociável da América Latina.

As respostas das entrevistas parecem apontar para uma escassa problematização da América Latina na formação de professores de espanhol como uma rica fonte de conhecimento. Sobre a origem do termo há uma grande controvérsia. Para alguns, a expressão América Latina tem como origem o poema do ensaísta colombiano José Maria Torres Caicedo, publicado em Paris (França), com o título de "Las dos Américas" ([...] La unión es su deber, su ley amarse: igual origen tienen y misión / La raza de la América latina, al frente tiene la sajona raza, enemiga mortal que ya amenaza su libertad destruir y su pendón [...]) no qual conclamava a união entre os povos da América Central e do Sul para preservação da liberdade e resistência contra a possível intervenção norte-americana na área.

\section{OS ELEMENTOS SÓCIO E INTERCULTURAIS NA FORMAÇÃO DO- CENTE}

A seleção da pluralidade cultural como um dos temas transversais registrados nos PCNs (Brasil, 2000) para ser incluído no contexto escolar incentivou o debate a respeito da configuração heterogênea que retrata a sociedade. Esse espaço de convivência no qual pessoas de diversas culturas não somente coexistem lado a lado mas se inter-relacionam levou para dentro da escola a discussão dessa temática a fim de fomentar o reconhecimento, respeito e valorização de grupos sociais menos valorizados.

O movimento do multiculturalismo advindo desses grupos sociais discriminados e excluídos e dos movimentos sociais que reivindicavam por igualdade e respeito nasceu fora do ambiente acadêmico como uma manifestação em busca de uma convivência democrática entre todas as culturas. Contudo, dentro do âmbito acadêmico, como opina Candau (2011, p.19), “[...] sua presença é frágil e objeto de muitas discussões [...] só recentemente (as questões relativas ao multiculturalismo) têm sido incluídas nos cursos de formação inicial de educadores/as e, assim mesmo, de modo esporádico e pouco sistemático, ao sabor de iniciativas pessoais de alguns VOL. 14 | N.2 | DEZ. 2017 
professores/as". Provavelmente, essa pouca penetração nos centros de ensino ocorra, por um lado, pela resistência de alguns docentes quanto à admissão da heterogeneidade cultural e consequentemente de diferentes formas de aprendizagem, o que exigiria do professor pesquisa e habilidade para viabilizar o conhecimento à todos; por outro lado, essa obstaculização justifica-se pela luta de um grupo de professores em manter os padrões de ensino da classe dominante.

Enquanto objeto de pesquisa, a formação docente passou a ser problematizada a partir da década de 90 com o propósito de "[...] superar a visão estática, conteudista, limitada ao domínio de métodos e técnicas de ensino ainda presente na formulação de cursos e de outras atividades de mesma natureza" (GOMES, 2011, p. 13). Nessa perspectiva, observou-se que questões do cotidiano não eram incluídas nas matrizes curriculares dos curso de formação, como por exemplo, a diversidade cultural. A notável ausência desse assunto deixou um profundo lapso na educação brasileira que não pensou na diferença como o ponto-chave das ações pedagógicas, a força-motriz para a construção de uma competência relevante a ser praticada por todos os educadores.

E é nesse contexto de convivência democrática entre culturas distintas que irrompe o conceito de interculturalidade. Diferentemente do multiculturalismo que reconhece as diversas culturas conviventes em um mesmo espaço e, por isso, se recusa a considerar uma única cultura como o centro, a interculturalidade abarca a política de convivência e fomenta a aproximação entre essas culturas resguardando, cada uma, as suas particularidades. Dessa maneira, não é suficiente assentir a existência do 'outro', mas procurar identificar os aspectos que coincidem entre a nossa cultura e a do 'outro' e com isso detectar as particularidades que nos individualizam e nos fazem reconhecer pertencentes a um determinado grupo. $\mathrm{O}$ reconhecimento dessas características deve conduzir ao respeito com relação à cultura particular para uma convivência pacífica, diferentemente da tolerância que somente 'aceita' as diferenças, porém não viabiliza a interação.

Para Fleuri (2003, p.17), "trata-se, na realidade, de um novo ponto de vista baseado no respeito à diferença, que se concretiza no reconhecimento da paridade de direitos". Nesse delineamento, procura-se aproximar as características de cada cultura, sem pretender anulá-las, usando-as como fonte de enriquecimento cultural.

No âmbito do ensino do espanhol, de acordo com a análise dos questionários, os elementos socioculturais não são trabalhados na perspectiva intercultural. De acordo com os professores investigados, as culturas dos países hispânicos são apresentadas por meio de recursos audiovisuais ou então através da apresentação de trabalhos de pesquisa realizadas pelos próprios aprendizes sobre alguns países hispanófonos. Essa prática de ensino possibilita, quando muito, apenas o conhecimento do grupo envolvido com os aspectos culturais do país pesquisado, não viabiliza a identificação de semelhanças e contrastes entre as diferentes culturas e tampouco aproxima a cultura brasileira das culturas hispânicas.

Pensamos, inclusive, que a construção da identidade cultural latino-americana do Brasil, enquanto única nação luso-falante no continente sul-americano, se tornaria mais viável em uma formação docente inicial assentada sob a ótica da interculturalidade. A identidade, afirma Hall (2016, p.21), diz "quem somos e a quem 'pertencemos"', distingue as semelhanças e as diferenças entre 'nós'e 'os outros'.

Nesse caminho, a abordagem intercultural exige do professor a prática de pesquisa para a construção de pontes entre as diversas culturas.

\section{O PROFESSOR COMO PESQUISADOR}

Inegavelmente, a globalização enquanto movimento de integração das economias deu novas diretrizes ao cenário mundial e exigiu profissionais mais bem qualificados para estabelecer relações comerciais, econômicas e políticas com os mercados de outros países. Com a ruptura das barreiras geográficas, os padrões in- 
A educação, como não poderia deixar de ser, também se viu convocada a ajustar-se à essa nova configuração educacional que requeria profissionais habilidosos e multifacetados para promover as transformações necessárias no ensino escolar. Nessa conjuntura complexa, as práticas educativas também se tornavam mais complexas e uma redefinição foi necessária, sobretudo nos cursos de formação inicial e continuada de professores. Dessa maneira, a ressignificação do ensino tornou-se prioridade. No formato de educação pensado para o século XXI não se concebia mais professores apenas como reprodutores do conhecimento produzido por pesquisadores externos.

Um dos caminhos apontados para viabilizar a melhoria na educação seria começar pelos processos formativos de professores. Para isso, os cursos de formação inicial deveriam fomentar a pesquisa como atividade imprescindível para o professor na contemporaneidade a fim de incentivá-los a produzir novos conhecimentos e atualizar os já existentes. Do ponto de vista de Veiga,

o papel da pesquisa na formação do professor não é enfatizado com relevância. Sem ser um pesquisador, resta ao professor a tarefa de aplicar métodos e técnicas, reproduzir e transmitir conhecimentos. A instituição formadora se resumiria a um centro de divulgação de conhecimentos produzidos por outros. (VEIGA, 2012, p.74-75)

Para a autora, os cursos de formação inicial deveriam ser o espaço apropriado para o desenvolvimento de pesquisas com caráter acadêmico e científico de forma a não dissociar o ensino da investigação. Essa desvinculação entre as duas atividades faz do professor um mero profissional sem o senso crítico, descompromissado com a prática reflexiva e, portanto, sem perspectiva de inovação da prática educativa em sala de aula. Alijado do processo de construção do conhecimento, ao professor resta-lhe somente a tarefa de repassar os conteúdos escolares. E não sendo formados com essa concepção, em sua prática educacional tampouco desperta no aluno o interesse pela investigação.

Em nossas análises, contatamos que, para alguns professores, o desenvolvimento de pesquisa está associada à inserção e participação em grupos de pesquisas criados e instituídos pelas universidades que se apoiam nas atividades de ensino, pesquisa e extensão. Para outros, ela não ocorre como deveria pela 'falta de tempo' em virtude da sobrecarga de trabalho. No entanto, é consensual a necessidade constante de realizá-la para atualização do conhecimento e melhoria na qualidade do ensino.

Aliás, é por meio da pesquisa, defende Tardif (s.d), que o princípio formativo se entrecruza com a profissão docente. Esse processo investigativo é capaz de fornecer um conhecimento fundamentado como resultado da análise interpretativa dos dados advindos da prática e do trabalho pedagógico e com isso, possivelmente, encontrar respostas para as difíceis situações postas pela prática social. Nesse sentido, reduz-se a polarização entre pesquisador e docentes tão fortemente marcada no processo educacional de modo a despertar o senso investigativo no professor que passa a ser um professor-pesquisador. (TARDIF et al, s.d).

\section{CONSIDERAÇÕES FINAIS}

O estudo detalhado dos Projetos Pedagógicos das três instituições de ensino superior analisadas que ofertam a Licenciatura em Língua Espanhola revelou que os desenhos curriculares dos cursos de formação inicial não contemplam a diversidade cultural hispanófona na formação do professor para essa área de atuação. Apenas a ESMAC, entre os seus componentes curriculares, abre espaço para a discussão da multiculturalidade ainda assim com uma carga horária pequena e obedecendo a determinação da Resolução CNE/CP n 01 de junho de 2004 que guia as Diretrizes Curriculares Nacionais para a Educação das Relações Étnico-Raciais e para o Ensino 
de História e Cultura Afro-Brasileira, Africana e Indígena.

Com isso, confirmou-se a hipótese de que há uma dissociação entre o ensino dos aspectos linguísticos e as práticas culturais dos países hispanófonos. Os cursos de formação docente inicial têm características majoritariamente metalinguageiros provavelmente por uma exigência mercadológica de formar conhecedores da gramática normativa de espanhol para atuar na educação básica onde as aulas de línguas, materna ou estrangeira, viraram sinônimo de ensino de gramática.

Pensamos que uma formação docente que aborde a diversidade cultural hispanófona contribuiria para desmistificar a crença na superioridade de uma cultura sobre as outras e levaria o estudante a conhecer as culturas de grande parte dos países hispanos que pouco são problematizados em sala de aula. Geralmente, a cultura e a variedade linguística peninsular ganham mais visibilidade que os países latino-americanos.

Por meio de uma abordagem intercultural das culturas hispanófonas seria possível encontrar semelhanças e distanciamento entre as culturas materna e estrangeira, o que contribuiria para superar os preconceitos erguidos, favorecer a mobilidade entre indivíduos de diferentes nacionalidade e propiciar uma convivência harmônica entre os povos.

A docência, de modo geral, exige capacitação própria e em se tratando do ensino de línguas há algumas especificidades em particular. Do professor exige-se não apenas o domínio do código linguístico da língua alvo como também dos aspectos socioculturais do povo que fala essa língua; requere a constante atualização com as metodologias de ensino mais modernas aplicadas no exterior voltadas para essa área de atuação; cobra a ação reflexiva e o pensamento crítico da fundamentação teórica. Não obstante, o saber pedagógico também é de relevante necessidade para esse professor que ensina a língua e a cultura do 'outro'.

Em resumo, desse professor espera-se "o desenvolvimento de sua sensibilidade intercultural, já que, mais que nenhum outro, o professor de língua estrangeira, como ponte de diferentes culturas" (VOLPI, 2008, p. 140), distingue-se pelo olhar diferenciado em direção às identidades, convicções e estilos de vida dos diferentes grupos.

Dos cursos de formação para professores de espanhol como língua estrangeira ressalta-se a enorme necessidade de repensar os processos formativos

\section{REFERÊNCIAS}

BRASIL. Lei ${ }^{\circ} 11.161$, de 05 de agosto de 2005. Dispõe sobre o ensino da língua espanhola. Disponível em: < http://www.planalto.gov.br/ccivil_03/_ato20042006/2005/ lei/111161.htm >. Acesso em: 04 set. 2016.

Parâmetros curriculares nacionais: ensino médio. Linguagens, códigos e suas tecnologias. p.25-32. Brasília: Ministério da Educação/ Secretaria de Educação Média e Tecnológica, 2000. Disponível em: <http://portal.mec.gov.br/seb/ arquivos/pdf/14_24.pdf > Acesso em: 10 mar. 2016.

CANDAU, Vera Maria. Multiculturalismo e educação: desafios para a prática pedagógica. In: MOREIRA, Antônio Flávio Barbosa; CANDAU, Vera Maria (org.). Multiculturalismo: diferenças culturais e práticas pedagógicas. 7. ed. Petrópolis, RJ: Vozes, 2011, p.13-37.

FLEURI, Reinaldo Matias. Intercultura e educação. Revista Brasileira de Educação, n. 23, maio-ago. 2003, p.16-35. Disponível em: <http://www.scielo.br/pdf/rbedu/n23/n23a02> Acesso em: 15 maio 2016.

GOMES, Nilma Lino; SILVA, Petronilha Beatriz Gonçalves e. O desafio da diversi- 
de professores. 3. ed. Belo Horizonte: Autêntica Editora, 2011, p.11-26.

IRINEU, Lucineudo Machado. Memórias sobre a América Latina na formação de professores de espanhol. In: LIMA, Lucielena Mendonça de. A (In) visibilidade da América Latina na formação do professor de espanhol. Coleção: Novas perspectivas em linguística aplicada. vol. 34. Campinas-SP: Pontes editores, 2014, p.21-39.

PRADO, Maria Lígia; PELLEGRINO, Gabriela. História da América Latina. São Paulo: Contexto, 2016.

SANTOS, Edleise Mendes Oliveira. Abordagem comunicativa intercultural (ACIN): uma proposta para ensinar e aprender língua no diálogo de culturas. Campinas, S.P: UNICAMP, 2004. 440 p. Tese (Doutorado). Disponível em: < file://C:/Users/ Ana\%20Paula/Downloads/Santos_EdleiseMendesOliveira_D.pdf $>$ Acesso em: 14 dez. 2017.

TARDIF, Maurice; LESSARD, Claude; GAUTHIER, Clermont. Formação de professores e contextos sociais: perspectivas internacionais. Porto: Rés Editora, s.d.

VEIGA, Ilma Passos Alencastro. Professor: tecnólogo do ensino ou agente social? In: _ _ AMARAL, Ana Lúcia (Orgs.). Formação de professores: políticas e debates. 5. ed. Campinas, S.P: Papirus, 2012, p.61-86.

VOLPI, Marina Tazón. A formação de professores de lingua estrangeira frente aos novos enfoques de sua função docente. In: LEFFA, Vilson José. O professor de línguas estrangeiras: construindo a profissão. Pelotas, RS: EDUCAT, 2008, p.133-141. 